Journal of Mathematics and Statistics 7 (1): 12-19, 2011

ISSN 1549-3644

(C) 2010 Science Publications

\title{
Periodic Review Probabilistic Multi-Item Inventory System with Zero Lead Time under Constraint and Varying Holding Cost
}

\author{
Naglaa Hassan El-Sodany \\ Third Statistician, General Department of National Accounts, \\ Central Agency for Public Mobilization and Statistics, Cairo, Egypt
}

\begin{abstract}
Problem statement: This study treats the probabilistic safety stock n-items inventory system having varying holding cost and zero lead-time subject to linear constraint. Approach: The expected total cost is composed of three components: the average purchase cost; the expected order cost and the expected holding cost. Results: The policy variables for this model are the number of periods $\mathrm{N}_{\mathrm{r}}^{*}$ and the optimal maximum inventory level $\mathrm{Q}_{\mathrm{mr}}^{*}$ and the minimum expected total cost.

Conclusion/Recommendations: We can obtain the optimal values of these policy variables by using the geometric programming approach. A special case is deduced and an illustrative numerical example is added.

Key words: Probabilistic safety stock multi-item, zero lead-time, varying holding cost, constrained probabilistic inventory system, random variable, demand fluctuations, geometric programming techniques, orthogonal conditions.
\end{abstract}

\section{INTRODUCTION}

In many situations demand is probabilistic since it is a random variable having a known probability distribution. All researchers have studied unconstrained probabilistic inventory models assuming the holding cost to be constant. Hadley and Within (1963) and Taha (1997) and Ben-Daya (1999) have examined unconstrained probabilistic inventory problems.

Fabrycky and Banks (1967) studied the probabilistic single-item, single source inventory system with zero lead-time, using the classical optimization. Also Hariri and Abou-El-Ata (1995; 1997) and Kotb (1998) investigated the constrained deterministic inventory models using a geometric programming approach. Recently, Abou-El-Ata (2002) and Fergany (2005) introduced the probabilistic multiitem inventory system with zero lead time under constraints and varying order cost, using geometric programming approach.

The aim of this study is to investigate the probabilistic safety stock multi-item, single source inventory model with zero lead-time and varying holding cost. The developed models are the probabilistic safety stock multi-item, single source inventory model with zero lead-time and varying holding cost under the expected order cost constraint and the probabilistic safety stock multi-item, single source inventory model with zero lead-time and varying holding cost under the expected varying holding cost constraint. The optimal numbers of periods $\mathrm{N}_{\mathrm{r}}^{*}$, the optimal maximum inventory levels $\mathrm{Q}_{\mathrm{mr}}^{*}$ and the minimum expected total cost are obtained. Also a special case is deduced and an illustrative numerical example is added.

Model development: The following notations are adopted for developing our model:

$\mathrm{c}_{\mathrm{pr}}=$ The purchase cost of the $\mathrm{r}^{\text {th }}$ item,

$\mathrm{c}_{\mathrm{or}} \quad=$ The order cost of the $\mathrm{r}^{\text {th }}$ item per cycle

$\mathrm{c}_{\mathrm{hr}}\left(\mathrm{N}_{\mathrm{r}}\right)=$ The varying holding cost of the $\mathrm{r}^{\text {th }}$ item per period, which takes the form

$\mathrm{C}_{\mathrm{hr}}\left(\mathrm{N}_{\mathrm{r}}\right)=\mathrm{c}_{\mathrm{hr}} \mathrm{N}_{\mathrm{r}}^{\beta}$

where, $c_{\mathrm{hr}}>0$ and $\beta>0$ are constant real numbers selected to provide us the best estimation of the cost function.

$\overline{\mathrm{H}}_{\mathrm{r}} \quad=$ The expected level of inventory held per $\mathrm{r}^{\text {th }}$ cycle

$\alpha \quad=$ The positive value representing apart of time for safety stock

$\mathrm{x}_{\mathrm{r}} \quad=\mathrm{A}$ random variable represent the demand of the $\mathrm{r}^{\text {th }}$ item during the cycle $\mathrm{N}_{\mathrm{r}}$

Corresponding Author: Naglaa, H. El-Sodany, Third Statistician, General Department of National Accounts, Central Agency for Public Mobilization and Statistics, Cairo, Egypt. 
$\mathrm{F}\left(\mathrm{x}_{\mathrm{r}}\right)=$ The probability density function of the demand $\mathrm{x}_{\mathrm{r}}$

$E\left(x_{r}\right)=$ The expected value of the demand

$$
x_{r}=\int_{X_{\text {ir }}}^{x_{\text {ir }}} x_{r} f\left(x_{r}\right) d x_{r}
$$

where, $\mathrm{x}_{\mathrm{ur}}$ and $\mathrm{x}_{\mathrm{lr}}$ are the maximum value and minimum value of the demand $x_{r}$, respectively

$D_{\mathrm{r}} \quad=$ The annual demand rate of the $\mathrm{r}^{\text {th }}$ item per period

$\mathrm{E}\left(\mathrm{D}_{\mathrm{r}}\right)=$ The expected annual demand $\mathrm{D}_{\mathrm{r}}$

$\mathrm{Q}_{\mathrm{mr}}=$ The maximum inventory level of the $\mathrm{r}^{\text {th }}$ item

$\mathrm{N}_{\mathrm{r}} \quad=$ The number of period, cycle, of the $\mathrm{r}^{\text {th }}$ item (a decision variable) and a review of the stock level of the $\mathrm{r}^{\text {th }}$ item is made every $\mathrm{N}_{\mathrm{r}}$ period

$\mathrm{K}_{\mathrm{o}}=$ The limitation on the expected order cost

$\mathrm{K}_{\mathrm{h}}=$ The limitation on the expected varying holding cost

$\mathrm{E}(\mathrm{PC})=$ The expected annual purchase cost

$\mathrm{E}(\mathrm{HC})=$ The expected annual holding cost

$\mathrm{E}(\mathrm{OC})=$ The expected annual ordering cost

$\mathrm{E}(\mathrm{TC})=$ The expected total cost function

The model analysis: consider an inventory process in which a review of the stock level is made every $\mathrm{N}_{\mathrm{r}}$ periods, $r=1,2, \ldots, n$. An amount is ordered so that the stock level is returned to its initial position designated by: $\mathrm{Q}_{\mathrm{mr}}, \mathrm{r}=1,2, \ldots, \mathrm{n}$. To avoid shortage during $\mathrm{N}_{\mathrm{r}}$ periods we must maintain a safety stock absorbing demand fluctuations. Also, this is done maintaining the quantity $\mathrm{Q}_{\mathrm{mr}}=\mathrm{x}_{\mathrm{ur}}$ for any cycle $\mathrm{N}_{\mathrm{r}}$. Hence the resulting safety stock, $E\left(D_{r}\right)$ a, meet the exceed demands cycle $N_{r}$. The system is represented graphically in Fig. 1.

The expected annual total cost is composed of three components: the expected purchase cost, the expected varying holding cost and the expected order cost, i.e.:

$\mathrm{E}(\mathrm{TC})=\mathrm{E}(\mathrm{PC})+\mathrm{E}(\mathrm{OC})+\mathrm{E}(\mathrm{HC})$

where the expected annual purchase cost is given by:

$$
\mathrm{E}(\mathrm{PC})=\sum_{\mathrm{r}=1}^{\mathrm{n}} \mathrm{c}_{\mathrm{pr}} \mathrm{E}\left(\mathrm{D}_{\mathrm{r}}\right)
$$

and the expected annual ordering cost is given by:

$$
\mathrm{E}(\mathrm{OC})=\sum_{\mathrm{r}=1}^{\mathrm{n}} \frac{\mathrm{c}_{\mathrm{or}}}{\mathrm{N}_{\mathrm{r}}}
$$

and the expected annual varying holding cost is given by:
$\mathrm{E}(\mathrm{HC})=\sum_{\mathrm{r}=1}^{\mathrm{n}} \frac{\mathrm{C}_{\mathrm{hr}}\left(\mathrm{N}_{\mathrm{r}}\right) \overline{\mathrm{H}}}{\mathrm{N}_{\mathrm{r}}}$

where, $\overline{\mathrm{H}}$ is the average inventory given by:

$$
\overline{\mathrm{H}}=\mathrm{N}_{\mathrm{r}}\left[\mathrm{Q}_{\mathrm{mr}}-\frac{\mathrm{E}\left(\mathrm{x}_{\mathrm{r}}\right)}{2}\right]
$$

Since, $\mathrm{E}\left(\mathrm{x}_{\mathrm{r}}\right)=\mathrm{E}(\mathrm{Dr}) \mathrm{N}_{\mathrm{r}}$, then:

$$
\overline{\mathrm{H}}=\mathrm{N}_{\mathrm{r}}\left[\mathrm{Q}_{\mathrm{mr}}-\frac{\mathrm{E}\left(\mathrm{D}_{\mathrm{r}}\right) \mathrm{N}_{\mathrm{r}}}{2}\right]
$$

The Optimization of the decision variables $\mathrm{N}_{\mathrm{r}}$ and $\mathrm{Q}_{\mathrm{mr}}$ can be performed if we assume that the maximum demand during the cycle, $\mathrm{x}_{\mathrm{ur}}$, is related to the expected demand during the cycle as:

$\mathrm{x}_{\mathrm{ur}}=\mathrm{E}\left(\mathrm{x}_{\mathrm{r}}\right) \mathrm{g}\left(\mathrm{N}_{\mathrm{r}}\right)=\mathrm{E}\left(\mathrm{D}_{\mathrm{r}}\right) \mathrm{N}_{\mathrm{r}} \mathrm{g}\left(\mathrm{N}_{\mathrm{r}}\right)$

where, $g\left(N_{r}\right)$ is a relational function, so we get:

$$
\overline{\mathrm{H}}_{\mathrm{r}}=\mathrm{E}\left(\mathrm{D}_{\mathrm{r}}\right) \mathrm{N}_{\mathrm{r}}^{2}\left[\frac{2 \mathrm{~g}\left(\mathrm{~N}_{\mathrm{r}}\right)-1}{2}\right]
$$

consider the case when $\mathrm{g}\left(\mathrm{N}_{\mathrm{r}}\right)$ is given by:

$$
g\left(N_{r}\right)=\frac{N_{r}+\alpha}{N_{r}}
$$

Then:

$\overline{\mathrm{H}}_{\mathrm{r}}=\mathrm{N}_{\mathrm{r}}\left[\frac{\mathrm{E}\left(\mathrm{D}_{\mathrm{r}}\right) \mathrm{N}_{\mathrm{r}}}{2}+\mathrm{E}\left(\mathrm{D}_{\mathrm{r}}\right) \alpha\right]$

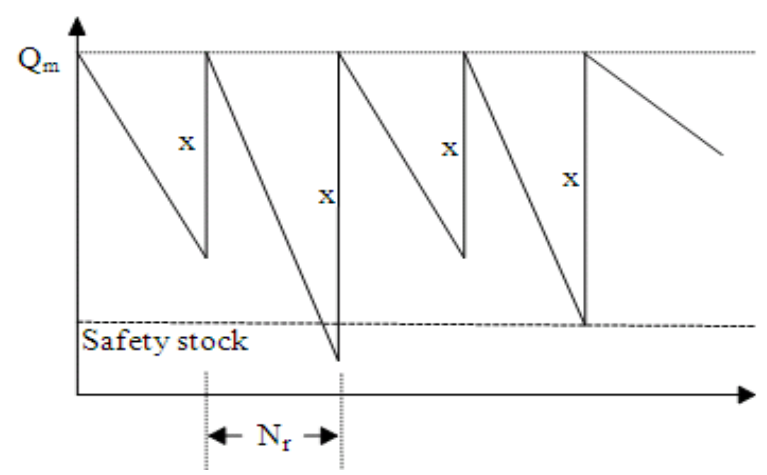

Fig. 1: Safety stock for periodic review inventory system 

by:

Then the expected varying holding cost is given

$$
\begin{aligned}
\mathrm{E}(\mathrm{HC}) & =\sum_{\mathrm{r}=1}^{\mathrm{n}}\left(\mathrm{C}_{\mathrm{hr}}\left(\mathrm{N}_{\mathrm{r}}\right) \frac{\mathrm{E}\left(\mathrm{D}_{\mathrm{r}}\right) \mathrm{N}_{\mathrm{r}}}{2}+\mathrm{c}_{\mathrm{hr}} \mathrm{E}\left(\mathrm{D}_{\mathrm{r}}\right) \alpha\right) \\
& =\sum_{\mathrm{r}=1}^{\mathrm{n}}\left(\frac{\mathrm{c}_{\mathrm{hr}} \mathrm{N}_{\mathrm{r}}^{\beta+1} \mathrm{E}\left(\mathrm{D}_{\mathrm{r}}\right)}{2}+\mathrm{c}_{\mathrm{hr}} \mathrm{E}\left(\mathrm{D}_{\mathrm{r}}\right) \alpha\right)
\end{aligned}
$$

where, $\mathrm{E}\left(\mathrm{D}_{\mathrm{r}}\right) \mathrm{a}$ is the safety stock required to absorb demand fluctuations during the inventory cycle $\mathrm{N}_{\mathrm{r}}$.

The expected total cost is then given by:

$$
\begin{aligned}
E(T C)= & \sum_{r=1}^{n}\left[c_{p r} E\left(D_{r}\right)+\frac{c_{o r}}{N_{r}}+\frac{c_{h r} N_{r}^{\beta+1} E\left(D_{r}\right)}{2}\right. \\
& \left.+c_{h r} E\left(D_{r}\right) \alpha\right]
\end{aligned}
$$

Our objective is to determine the optimal number of periods $\mathrm{N}_{\mathrm{r}}^{*}$ that minimize the expected total cost for the following two models:

Model (I): Safety stock for Probabilistic Periodic Review Multi- Item Inventory System with Zero Lead Time and Varying Holding Cost under the Expected Order Cost Limitation

Consider the relevant expected total cost (1), the restriction on the expected order cost is:

$\sum_{\mathrm{r}=1}^{\mathrm{n}} \frac{\mathrm{c}_{\mathrm{or}}}{\mathrm{N}_{\mathrm{r}}} \leq \mathrm{K}_{\mathrm{o}}$

The terms $\sum_{\mathrm{r}=1}^{\mathrm{n}} \mathrm{c}_{\mathrm{pr}} \mathrm{E}\left(\mathrm{D}_{\mathrm{r}}\right)$ and $\sum_{\mathrm{r}=1}^{\mathrm{n}} \mathrm{c}_{\mathrm{hr}} \mathrm{E}\left(\mathrm{D}_{\mathrm{r}}\right) \alpha$ are constants and can be postponed without any effect and then the expected total cost can be written as:

$$
\mathrm{E}(\mathrm{TC})=\sum_{\mathrm{r}=1}^{\mathrm{n}}\left[\frac{\mathrm{c}_{\mathrm{or}}}{\mathrm{N}_{\mathrm{r}}}+\frac{\mathrm{c}_{\mathrm{hr}} \mathrm{N}_{\mathrm{r}}^{\beta+1} \mathrm{E}\left(\mathrm{D}_{\mathrm{r}}\right)}{2}\right]
$$

Subject to:

$$
\sum_{\mathrm{r}=1}^{\mathrm{n}} \frac{\mathrm{c}_{\mathrm{or}}}{\mathrm{N}_{\mathrm{r}} \mathrm{K}_{\mathrm{o}}} \leq 1
$$

Applying the geometric programming techniques to Eqs.3 and 4, the enlarged predual function can be given by:

$$
\begin{aligned}
& G(\underline{W})=\prod_{r=1}^{n}\left(\frac{c_{o r}}{N_{r} W_{1 r}}\right)^{W_{1 r}}\left(\frac{c_{h r} N_{s+1}^{\beta+1} E\left(D_{r}\right)}{2 W_{2 r}}\right)^{W_{2 r}}\left(\frac{c_{o r}}{N_{r} k_{o} W_{3 r}}\right)^{W_{3 r}} \\
& =\prod_{\mathrm{r}=1}^{\mathrm{n}}\left(\frac{\mathrm{c}_{\mathrm{or}}}{\mathrm{W}_{1 \mathrm{r}}}\right)^{\mathrm{W}_{\mathrm{lr}}}\left(\frac{\mathrm{c}_{\mathrm{hr}} \mathrm{E}\left(\mathrm{D}_{\mathrm{r}}\right)}{2 \mathrm{~W}_{2 \mathrm{r}}}\right)^{\mathrm{W}_{2 \mathrm{r}}}\left(\frac{\mathrm{c}_{\mathrm{or}}}{\mathrm{k}_{\mathrm{o}} \mathrm{W}_{3 \mathrm{r}}}\right)^{\mathrm{W}_{3 \mathrm{r}}} \mathrm{N}_{\mathrm{r}}^{\mathrm{W}_{2 \mathrm{r}}(\beta+1)-\mathrm{W}_{\mathrm{Ir}}-\mathrm{W}_{3 \mathrm{r}}}
\end{aligned}
$$

where, $\underline{\mathrm{W}}=\mathrm{W}_{\mathrm{jr}}, 0<\mathrm{W}_{\mathrm{jr}}<1, \mathrm{j}=1,2,3, \mathrm{r}=1,2, \ldots ., \mathrm{n}$ are the weights and can be chosen to yield the normal and the orthogonal conditions as follows:

$\mathrm{W}_{1 \mathrm{r}}+\mathrm{W}_{2 \mathrm{r}}=1$
$\mathrm{~W}_{2 \mathrm{r}}(\beta+1)-\mathrm{W}_{1 \mathrm{r}}-\mathrm{W}_{3 \mathrm{r}}=0, \quad \mathrm{r}=1,2,3, \ldots, \mathrm{n}$

Solving the above equations, we get:

$\mathrm{W}_{1 \mathrm{r}}=\frac{\beta+1-\mathrm{W}_{3 \mathrm{r}}}{\beta+2} \quad$ and $\quad \mathrm{W}_{2 \mathrm{r}}=\frac{1+\mathrm{W}_{3 \mathrm{r}}}{\beta+2}$

Substituting from Eq.6 into Eq.5, the dual function is given in the form:

$$
g\left(W_{3 r}\right)=\prod_{r=1}^{n}\left(\frac{(\beta+2) c_{o r}}{\beta+1-W_{3 r}}\right)^{\frac{\beta+1-W_{3 r}}{\beta+2}}\left(\frac{c_{h r}(\beta+2) E\left(D_{r}\right)}{2\left(1+W_{3 r}\right)}\right)^{\frac{1+W_{3 r}}{\beta+2}}\left(\frac{c_{o r}}{k_{0} W_{3 r}}\right)^{W_{3 r}}
$$

Taking the logarithm of both sides of Eq. 7:

$$
\begin{aligned}
\operatorname{lng}\left(\mathrm{W}_{3 \mathrm{r}}\right) & =\sum_{\mathrm{r}=1}^{\mathrm{n}} \frac{\beta+1-\mathrm{W}_{3 \mathrm{r}}}{\beta+2}\left[\ln (\beta+2) \mathrm{c}_{\mathrm{or}}-\ln \left(\beta+1-\mathrm{W}_{3 \mathrm{r}}\right)\right] \\
& +\frac{1+\mathrm{W}_{3 \mathrm{r}}}{\beta+2}\left[\ln \mathrm{c}_{\mathrm{hr}}(\beta+2) \mathrm{E}\left(\mathrm{D}_{\mathrm{r}}\right)-\ln 2\left(1+\mathrm{W}_{3 \mathrm{r}}\right)\right] \\
& +\mathrm{w}_{3 \mathrm{r}}\left[\ln \frac{\mathrm{c}_{\mathrm{or}}}{\mathrm{K}_{\mathrm{o}}}-\ln \mathrm{W}_{3 \mathrm{r}}\right]
\end{aligned}
$$

To find the optimal value of $\mathrm{W}_{3 \mathrm{r}}$ which minimize $\mathrm{g}\left(\mathrm{W}_{3 \mathrm{r}}\right)$, take the first derivative of $\ln \mathrm{g}\left(\mathrm{W}_{3 \mathrm{r}}\right)$ with respect to $\mathrm{W}_{3 \mathrm{r}}$ and put it equal to zero, as follows:

$$
\begin{aligned}
\frac{\mathrm{d} \operatorname{lng}\left(\mathrm{W}_{3 \mathrm{r}}\right)}{\mathrm{dW}_{3 \mathrm{r}}}= & \frac{-1}{\beta+2}\left[\ln (\beta+2) \mathrm{c}_{\mathrm{or}}-\ln \left(\beta+1-\mathrm{W}_{3 \mathrm{r}}\right)\right] \\
& +\frac{1}{\beta+2}\left[\operatorname{lnc}_{\mathrm{hr}}(\beta+2) \mathrm{E}\left(\mathrm{D}_{\mathrm{r}}\right)-\ln 2\left(1+\mathrm{W}_{3 \mathrm{r}}\right)\right] \\
& +\ln \frac{\mathrm{c}_{\mathrm{or}}}{\mathrm{k}_{\mathrm{o}}}-\ln \mathrm{w}_{3 \mathrm{r}}-1=0
\end{aligned}
$$

Simplifying Eq. 8, we obtain:

$$
\begin{gathered}
f\left(W_{3 r}\right)=\frac{c_{h r} E\left(D_{r}\right)(\beta+1)}{2 c_{o r}}\left(\frac{c_{o r}}{K_{o} e}\right)^{\beta+2}-\frac{c_{h r} E\left(D_{r}\right) W_{3 r}}{2 c_{o r}}\left(\frac{c_{o r}}{K_{o} e}\right)^{\beta+2} \\
-\left(W_{3 r}^{\beta+3}+W_{3 r}^{\beta+3}\right)=0
\end{gathered}
$$

Let:

$$
A=\frac{c_{h r} E\left(D_{r}\right)}{2 c_{o r}}\left(\frac{c_{o r}}{K_{o} e}\right)^{\beta+2}
$$


Then, we obtain:

$\mathrm{f}\left(\mathrm{W}_{3 \mathrm{r}}\right)=\mathrm{W}_{3 \mathrm{r}}^{\beta+3}+\mathrm{W}_{3 \mathrm{r}}^{\beta+2}+\mathrm{AW}_{3 \mathrm{r}}-(\beta+1) \mathrm{A}=0$

Where:

$$
\begin{aligned}
& f(0)=-(\beta+1) A<0 \\
& f(1)=2-\beta A>0
\end{aligned}
$$

Which means that there exist a root $\mathrm{W}_{3 \mathrm{r}} \in(0,1)$. Any method such as the trial and error method could be used to calculate this root. However we shall first verify that $\mathrm{W}_{3 \mathrm{r}}{ }^{*}$ calculated from Eq. 9 maximize $\mathrm{g}\left(\mathrm{W}_{3 \mathrm{r}}\right)$. This is done by showing that the second derivative is always negative:

$$
\frac{\mathrm{d}^{2} \ln \mathrm{g}\left(\mathrm{W}_{3 \mathrm{r}}\right)}{\mathrm{dW_{3 \textrm {r } }}}=-\left[\begin{array}{c}
\frac{1}{(\beta+2)\left(\beta+1-\mathrm{W}_{3 \mathrm{r}}\right)}+ \\
\frac{1}{(\beta+2)\left(1+\mathrm{W}_{3 \mathrm{r}}\right)}+\frac{1}{\mathrm{~W}_{3 \mathrm{r}}}
\end{array}\right]<0
$$

Thus the root $\mathrm{W}_{3 \mathrm{r}}{ }^{*}$ calculated from Eq.9 maximize the dual function $\mathrm{g}\left(\mathrm{W}_{3 \mathrm{r}}\right)$. Hence the optimal solution is $\mathrm{W}_{\mathrm{jr}}{ }^{*}, \mathrm{j}=1,2,3$, where $\mathrm{W}_{3 \mathrm{r}}{ }^{*}$ is the solution of the Eq.9 and $\mathrm{W}_{1 \mathrm{r}}{ }^{*}, \mathrm{~W}_{2 \mathrm{r}}{ }^{*}$ are calculated by substituting the value of $\mathrm{W}_{3 \mathrm{r}}{ }^{*}$ in Eq. 6.

To find the optimal number of periods $\mathrm{N}_{\mathrm{r}}^{*}$, use the following relations due to Duffin and Peterson (1974) theorem as follows:

$$
\begin{aligned}
& \frac{\mathrm{c}_{\mathrm{or}}}{\mathrm{N}_{\mathrm{r}}^{*}}=\mathrm{W}_{1 \mathrm{r}}^{*} \mathrm{~g}\left(\mathrm{~W}_{3 \mathrm{r}}^{*}\right) \\
& \frac{\mathrm{c}_{\mathrm{hr}} \mathrm{N}_{\mathrm{r}}^{* \beta+1} \mathrm{E}\left(\mathrm{D}_{\mathrm{r}}\right)}{2}=\mathrm{W}_{2 \mathrm{r}}^{*} \mathrm{~g}\left(\mathrm{~W}_{3 \mathrm{r}}^{*}\right)
\end{aligned}
$$

Solving these equations, the optimal number of periods per cycle is given by:

$$
\mathrm{N}_{\mathrm{r}}^{*}=\left(\frac{2 \mathrm{c}_{\mathrm{or}}\left(1+\mathrm{W}_{3 \mathrm{r}}^{*}\right)}{\mathrm{c}_{\mathrm{hr}} \mathrm{E}\left(\mathrm{D}_{\mathrm{r}}\right)\left(\beta+1-\mathrm{W}_{3 \mathrm{r}}^{*}\right)}\right)^{\frac{1}{\beta+2}}
$$

Hence the optimal maximum inventory level is given by:

$$
\begin{aligned}
Q_{m r}^{*} & =E\left(D_{r}\right) N_{r}^{*}\left(\frac{N_{r}^{*}+\alpha}{N_{r}^{*}}\right) \\
& =E\left(D_{r}\right)\left(\frac{2 c_{o r}\left(1+W_{3 r}^{*}\right)}{c_{h r} E\left(D_{r}\right)\left(\beta+1-W_{3 r}^{*}\right)}\right)^{\frac{1}{\beta+2}}+E\left(D_{r}\right) \alpha
\end{aligned}
$$

Substituting the value of $\mathrm{N}_{\mathrm{r}}^{*}$ in Eq.3 after adding the constant terms, we get:

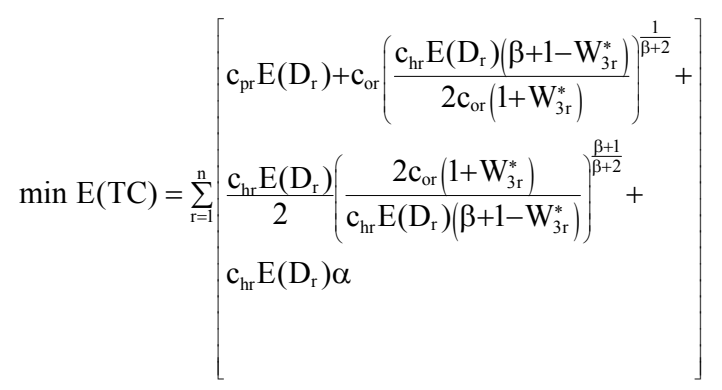

Model(II): Safety stock for Probabilistic Periodic Review Multi- Item Inventory System with Zero Lead Time and Varying Holding Cost under the Expected Varying Holding Cost Limitation

Consider the relevant expected total cost (1), the restriction on the expected varying holding cost is:

$\sum_{\mathrm{r}=1}^{\mathrm{n}} \frac{\mathrm{c}_{\mathrm{hr}} \mathrm{N}_{\mathrm{r}}^{\beta+1} \mathrm{E}\left(\mathrm{D}_{\mathrm{r}}\right)}{2} \leq \mathrm{K}_{\mathrm{h}}$

The terms $\sum_{\mathrm{r}=1}^{\mathrm{n}} \mathrm{c}_{\mathrm{pr}} \mathrm{E}\left(\mathrm{D}_{\mathrm{r}}\right)$ and $\sum_{\mathrm{r}=1}^{\mathrm{n}} \mathrm{c}_{\mathrm{hr}} \mathrm{E}\left(\mathrm{D}_{\mathrm{r}}\right) \alpha$ are constants and can be postponed without any effect and then the expected total cost can be written as:

$\mathrm{E}(\mathrm{TC})=\sum_{\mathrm{r}=1}^{\mathrm{n}}\left[\frac{\mathrm{c}_{\mathrm{or}}}{\mathrm{N}_{\mathrm{r}}}+\frac{\mathrm{c}_{\mathrm{hr}} \mathrm{N}_{\mathrm{r}}^{\beta+1} \mathrm{E}\left(\mathrm{D}_{\mathrm{r}}\right)}{2}\right]$

Subject to:

$\sum_{\mathrm{r}=1}^{\mathrm{n}} \frac{\mathrm{c}_{\mathrm{hr}} \mathrm{N}_{\mathrm{r}}^{\beta+1} \mathrm{E}\left(\mathrm{D}_{\mathrm{r}}\right)}{2 \mathrm{~K}_{\mathrm{h}}} \leq 1$

Applying the geometric programming techniques to Eq.13 and 14, the enlarged predual function can be given by:

$$
\begin{aligned}
& \mathrm{G}(\underline{\mathrm{W}})=\prod_{\mathrm{r}=1}^{\mathrm{n}}\left(\frac{\mathrm{c}_{\mathrm{or}}}{\mathrm{N}_{\mathrm{r}} \mathrm{W}_{\mathrm{lr}}}\right)^{\mathrm{W}_{\mathrm{rr}}}\left(\frac{\mathrm{c}_{\mathrm{hr}} \mathrm{N}_{\mathrm{r}}^{\beta+1} \mathrm{E}\left(\mathrm{D}_{\mathrm{r}}\right)}{2 \mathrm{~W}_{2 \mathrm{r}}}\right)^{\mathrm{W}_{2 \mathrm{r}}}\left(\frac{\mathrm{c}_{\mathrm{hr}} \mathrm{N}_{\mathrm{r}}^{\beta+1} \mathrm{E}\left(\mathrm{D}_{\mathrm{r}}\right)}{2 \mathrm{k}_{\mathrm{h}} \mathrm{W}_{3 \mathrm{r}}}\right)^{\mathrm{W}_{3 \mathrm{r}}} \\
& =\prod_{r=1}^{n}\left(\frac{c_{o r}}{W_{l r}}\right)^{W_{1 r}}\left(\frac{c_{h r} E\left(D_{r}\right)}{2 W_{2 r}}\right)^{W_{2 r}}\left(\frac{c_{h r} E\left(D_{r}\right)}{2 k_{h} W_{3 r}}\right)^{W_{3 r}} N_{r}^{\left(W_{2 r}+W_{3 r}\right)(\beta+1)-W_{l r}}
\end{aligned}
$$

where, $\underline{\mathrm{W}}=\mathrm{W}_{\mathrm{jr}}, 0<\mathrm{W}_{\mathrm{jr}}<1, \mathrm{j}=1,2,3, \mathrm{r}=1,2, \ldots ., \mathrm{n}$ are the weights and can be chosen to yield the normal and the orthogonal conditions as follows: 
$\mathrm{W}_{1 \mathrm{r}}+\mathrm{W}_{2 \mathrm{r}}=1$

$\left(\mathrm{W}_{2 \mathrm{r}}+\mathrm{W}_{3 \mathrm{r}}\right)(\beta+1)-\mathrm{W}_{\mathrm{lr}}=0, \quad \mathrm{r}=1,2,3, \ldots, \mathrm{n}$

Solving the above equations, we get:

$\mathrm{W}_{\mathrm{lr}}=\frac{(\beta+1)\left(1+\mathrm{W}_{3 \mathrm{r}}\right)}{\beta+2}$ and $\mathrm{W}_{2 \mathrm{r}}=\frac{1-(\beta+1) \mathrm{W}_{3 \mathrm{r}}}{\beta+2}$

Substituting from Eq. 16 into Eq. 15, the dual function is given in the form:

$$
\begin{aligned}
g\left(W_{3 r}\right)= & \prod_{\mathrm{r}=1}^{\mathrm{n}}\left(\frac{(\beta+2) \mathrm{c}_{\text {or }}}{\left((\beta+1)\left(1+\mathrm{W}_{3 \mathrm{r}}\right)\right.}\right)^{\frac{(\beta+1)\left(1+\mathrm{W}_{3 \mathrm{r}}\right)}{\beta+2}}\left(\frac{\mathrm{c}_{\mathrm{hr}}(\beta+2) \mathrm{E}\left(\mathrm{D}_{\mathrm{r}}\right)}{2\left(1-(\beta+1) \mathrm{W}_{3 \mathrm{r}}\right)}\right)^{\frac{1-(\beta+1) \mathrm{W}_{3 \mathrm{r}}}{\beta+2}} \\
& \left(\frac{\mathrm{c}_{\mathrm{hr}} \mathrm{E}\left(\mathrm{D}_{\mathrm{r}}\right)}{2 \mathrm{~K}_{\mathrm{h}} \mathrm{W}_{3 \mathrm{r}}}\right)^{\mathrm{W}_{3 \mathrm{r}}}
\end{aligned}
$$

Taking the logarithm of both sides of Eq.17:

$$
\begin{aligned}
& \operatorname{lng}\left(\mathrm{W}_{3 \mathrm{r}}\right)=\sum_{\mathrm{r}=1}^{\mathrm{n}} \frac{(\beta+1)\left(1+\mathrm{W}_{3 \mathrm{r}}\right)}{\beta+2}\left[\ln (\beta+2) \mathrm{c}_{\mathrm{or}}-\ln (\beta+1)\left(1+\mathrm{W}_{3 \mathrm{r}}\right)\right] \\
& +\frac{1-(\beta+1) \mathrm{W}_{3 \mathrm{r}}}{\beta+2}\left[\operatorname{lnc}_{\mathrm{hr}} \mathrm{E}\left(\mathrm{D}_{\mathrm{r}}\right)(\beta+2)-\ln 2\left(1-(\beta+1) \mathrm{W}_{3 \mathrm{r}}\right)\right] \\
& +\mathrm{W}_{3 \mathrm{r}}\left[\ln \frac{\mathrm{c}_{\mathrm{hr}} \mathrm{E}\left(\mathrm{D}_{\mathrm{r}}\right)}{2 \mathrm{~K}_{\mathrm{h}}}-\ln \mathrm{W}_{3 \mathrm{r}}\right]
\end{aligned}
$$

To find the optimal value of $\mathrm{W}_{3 \mathrm{r}}$ which minimize $\mathrm{g}\left(\mathrm{W}_{3 \mathrm{r}}\right)$, take the first derivative of $\ln \mathrm{g}\left(\mathrm{W}_{3 \mathrm{r}}\right)$ with respect to $\mathrm{W}_{3 \mathrm{r}}$ and put it equal to zero, as follows:

$$
\begin{aligned}
\frac{\mathrm{d} \ln \mathrm{g}\left(\mathrm{W}_{3 \mathrm{r}}\right)}{\mathrm{dW}_{3 \mathrm{r}}}=\frac{\beta+1}{\beta+2}\left[\ln (\beta+2) \mathrm{c}_{\mathrm{or}}-\ln (\beta+1)\left(1+\mathrm{W}_{3 \mathrm{r}}\right)\right] \\
\quad-\frac{\beta+1}{\beta+2}\left[\ln \mathrm{c}_{\mathrm{hr}} \mathrm{E}\left(\mathrm{D}_{\mathrm{r}}\right)(\beta+2)-\ln 2\left(1-(\beta+1) \mathrm{W}_{3 \mathrm{r}}\right)\right] \\
+\ln \frac{\mathrm{c}_{\mathrm{hr}} \mathrm{E}\left(\mathrm{D}_{\mathrm{r}}\right)}{2 \mathrm{~K}_{\mathrm{h}}}-\ln \mathrm{W}_{3 \mathrm{r}}-1=0
\end{aligned}
$$

Simplifying Eq. 18, we obtain:

$$
\begin{aligned}
f\left(W_{3 r}\right)= & W_{3 r}^{\frac{\beta+2}{\beta+1}}\left(1+W_{3 r}\right)+\frac{2 c_{o r} W_{3 r}}{c_{h r} E\left(D_{r}\right)}\left(\frac{c_{h r} E\left(D_{r}\right)}{2 K_{h} e}\right)^{\frac{\beta+2}{\beta+1}} \\
& -\frac{2 c_{o r}}{(\beta+1) c_{h r} E\left(D_{r}\right)}\left(\frac{c_{h r} E\left(D_{r}\right)}{2 K_{h} e}\right)^{\frac{\beta+2}{\beta+1}}=0
\end{aligned}
$$

Let:

$$
A=\frac{2 c_{o r}}{(\beta+1) c_{h r} E\left(D_{r}\right)}\left(\frac{c_{h r} E\left(D_{r}\right)}{2 K_{h} \mathrm{e}}\right)^{\frac{\beta+2}{\beta+1}}
$$

Then, we obtain:

$\mathrm{f}\left(\mathrm{W}_{3 \mathrm{r}}\right)=\mathrm{W}_{3 \mathrm{r}}^{\frac{2 \beta+3}{\beta+1}}+\mathrm{W}_{3 \mathrm{r}}^{\frac{\beta+2}{\beta+1}}+\mathrm{A}(\beta+1) \mathrm{W}_{3 \mathrm{r}}-\mathrm{A}=0$

Where:

$\mathrm{f}(0)=-\mathrm{A}<0$
$\mathrm{f}(1)=2+\mathrm{A} \beta>0$

Which means that there exist a root $\mathrm{W}_{3 \mathrm{r}} \in(0,1)$. Any method such as the trial and error method could be used to calculate this root. However we shall first verify that $\mathrm{W}_{3 \mathrm{r}}{ }^{*}$ calculated from Eq. 19 maximize $\mathrm{g}\left(\mathrm{W}_{3 \mathrm{r}}\right)$. This is done by showing that the second derivative is always negative:

$$
\frac{\mathrm{d}^{2} \ln \mathrm{g}\left(\mathrm{W}_{3 \mathrm{r}}\right)}{\mathrm{dW}_{3 \mathrm{r}}^{2}}=-\left[\begin{array}{c}
\frac{(\beta+1)}{(\beta+2)\left(1+\mathrm{W}_{3 \mathrm{r}}\right)}+\frac{(\beta+1)^{2}}{(\beta+2)\left(1-(\beta+1) \mathrm{W}_{3 \mathrm{r}}\right)} \\
+\frac{1}{\mathrm{~W}_{3 \mathrm{r}}}
\end{array}\right]<0
$$

Thus the root $\mathrm{W}_{3 \mathrm{r}}{ }^{*}$ calculated from (19) maximize the dual function $g\left(\mathrm{~W}_{3 \mathrm{r}}\right)$. Hence the optimal solution is $\mathrm{W}_{\mathrm{jr}}{ }^{*}, \mathrm{j}=1,2,3$, where $\mathrm{W}_{3 \mathrm{r}}{ }^{*}$ is the solution of (19) and $\mathrm{W}_{\mathrm{lr}}{ }^{*}, \mathrm{~W}_{2 \mathrm{r}}{ }^{*}$ are calculated by substituting the value of $\mathrm{W}_{3 \mathrm{r}}{ }^{*}$ in Eq.16.

To find the optimal number of periods $\mathrm{N}_{\mathrm{r}}^{*}$, use the following relations due to Duffin and Peterson (1974) theorem as follows:

$\frac{\mathrm{c}_{\mathrm{or}}}{\mathrm{N}_{\mathrm{r}}{ }^{*}}=\mathrm{W}_{1 \mathrm{r}}{ }^{*} \mathrm{~g}\left(\mathrm{~W}_{3 \mathrm{r}}{ }^{*}\right)$

$\frac{\mathrm{c}_{\mathrm{hr}} \mathrm{N}_{\mathrm{r}}{ }^{*}+1 \mathrm{E}\left(\mathrm{D}_{\mathrm{r}}\right)}{2}=\mathrm{W}_{2 \mathrm{r}}{ }^{*} \mathrm{~g}\left(\mathrm{~W}_{3 \mathrm{r}}{ }^{*}\right)$

Solving these equations, the optimal expected number of periods per cycle is given by:

$$
\mathrm{N}_{\mathrm{r}}^{*}=\left[\frac{2 \mathrm{c}_{\mathrm{or}}\left(1-(\beta+1) \mathrm{W}_{3 \mathrm{r}}\right)}{\mathrm{c}_{\mathrm{hr}} \mathrm{E}\left(\mathrm{D}_{\mathrm{r}}\right)(\beta+1)\left(1+\mathrm{W}_{3 \mathrm{r}}\right)}\right]^{\frac{1}{\beta+2}}
$$

Hence the optimal maximum inventory level is given by:

$\mathrm{Q}_{\mathrm{mr}}^{*}=\mathrm{E}\left(\mathrm{D}_{\mathrm{r}}\right)\left[\frac{2 \mathrm{c}_{\mathrm{or}}\left(1-(\beta+1) \mathrm{W}_{3 \mathrm{r}}\right)}{\mathrm{c}_{\mathrm{hr}} \mathrm{E}\left(\mathrm{D}_{\mathrm{r}}\right)(\beta+1)\left(1+\mathrm{W}_{3 \mathrm{r}}\right)}\right]^{\frac{1}{\beta+2}}+\mathrm{E}\left(\mathrm{D}_{\mathrm{r}}\right) \alpha$ 
Table 1: the parameters of the three items

\begin{tabular}{lrrr}
\hline Item parameters & Item 1 & Item 2 & Item 3 \\
\hline $\mathrm{E}\left(\mathrm{D}_{\mathrm{r}}\right)$ & 32.00 & 25.00 & 18.00 \\
$\mathrm{c}_{\mathrm{hr}}$ & 0.20 & 0.22 & 0.24 \\
$\mathrm{c}_{\mathrm{or}}$ & 150.00 & 170.00 & 190.00 \\
$\mathrm{c}_{\mathrm{pr}}$ & 100.00 & 120.00 & 140.00 \\
\hline
\end{tabular}

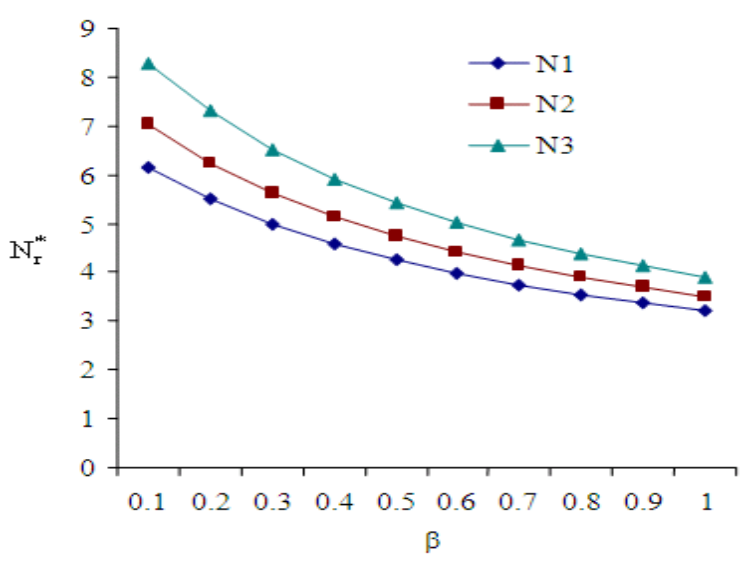

Fig. 2: The relation between $N_{r}^{*}$ and $\beta, K_{o}=200$

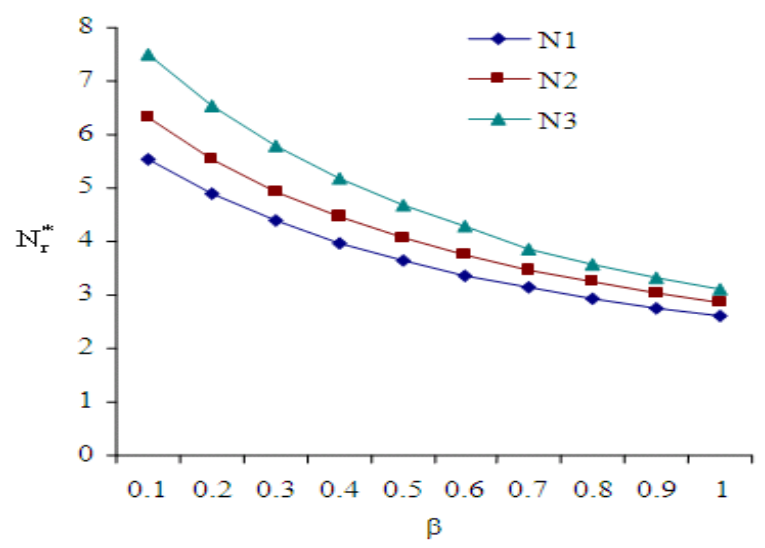

Fig. 3: The relation between $N_{r}^{*}$ and $\beta, K_{h}=100$

Substituting the value of $\mathrm{N}_{\mathrm{r}}^{*}$ in Eq. 13 after adding the constant term, we get:

$$
\min E(T C)=\sum_{r=1}^{n}\left[\begin{array}{l}
c_{p r} E\left(D_{r}\right)+c_{o r}\left[\frac{c_{h r} E\left(D_{r}\right)(\beta+1)\left(1+W_{3 r} r\right.}{2 c_{o r}\left(1-(\beta+1) W_{3 r}\right)}\right]^{\frac{1}{\beta+2}} \\
+\frac{c_{h r} E\left(D_{r}\right)}{2}\left[\frac{2 c_{o r}\left(1-(\beta+1) W_{3 r}\right)}{c_{h r} E\left(D_{r}\right)(\beta+1)\left(1+W_{3 r}\right)}\right]^{\frac{\beta+1}{\beta+2}} \\
+c_{h r} E\left(D_{r}\right) \alpha
\end{array}\right]
$$

Special case: We deduce a special case of our models as follows.

For Model (I), let $\beta=0, r=1$ and $K_{0} \rightarrow \infty$ then $\mathrm{C}_{\mathrm{hr}}\left(\mathrm{N}_{\mathrm{r}}\right)=\mathrm{c}_{\mathrm{hr}}$ and $\mathrm{W}_{3 \mathrm{r}}{ }^{*} \rightarrow 0$. Also, for Model (II), let $\beta=$ $0, \mathrm{r}=1$ and $\mathrm{K}_{\mathrm{h}} \rightarrow \infty$ then $\mathrm{C}_{\mathrm{hr}}\left(\mathrm{N}_{\mathrm{r}}\right)=\mathrm{c}_{\mathrm{hr}}$ and $\mathrm{W}_{3 \mathrm{r}}{ }^{*} \rightarrow 0$. Then Eq. 10 and 20 become:

$$
\mathrm{N}^{*}=\sqrt{\frac{2 \mathrm{c}_{\mathrm{o}}}{\mathrm{c}_{\mathrm{h}} \mathrm{E}(\mathrm{D})}}
$$

Also, Eq. 11 and 21 become:

$$
\mathrm{Q}_{\mathrm{m}}^{*}=\mathrm{E}(\mathrm{D}) \sqrt{\frac{2 \mathrm{c}_{\mathrm{o}}}{\mathrm{c}_{\mathrm{h}} \mathrm{E}(\mathrm{D})}}+\mathrm{E}(\mathrm{D}) \alpha
$$

Also, Eq. 12 and 22 become:

$\min \mathrm{E}(\mathrm{TC})=\mathrm{c}_{\mathrm{p}} \mathrm{E}(\mathrm{D})+\sqrt{2 \mathrm{c}_{\mathrm{o}} \mathrm{c}_{\mathrm{h}} \mathrm{E}(\mathrm{D})}+\mathrm{c}_{\mathrm{h}} \mathrm{E}(\mathrm{D}) \alpha$

This is a probabilistic single-item inventory model without any restriction and constant costs, which agree with the model of maintaining stock to absorb demand fluctions (Fabrycky and Banks, 1967)

An illustrative example: Consider the inventory parameters given in Table 1, we will find the optimal inventory doctrine by determining the minimum expected total cost when:

- The system is probabilistic periodic review multiitem inventory system under the expected order limitation $\mathrm{K}_{\mathrm{o}}=200$

- The system is probabilistic periodic review multiitem inventory system under the expected varying holding cost limitation $\mathrm{K}_{\mathrm{h}}=100$

Also assume that $\mathrm{a}=5$ and $0.1 \leq \beta \leq 1$.

Using the results of our models, the optimal expected number of periods per cycle, the optimal maximum inventory level and the minimum expected total cost min $\mathrm{E}(\mathrm{TC})$ can be summarized in the following Table 2 and 3.

The solution of the problem may be determined more readily by plotting min $\mathrm{E}(\mathrm{TC})$ against $\beta$ and $\mathrm{N}_{\mathrm{r}}^{*}$ against $\beta$ for the two inventory models in the following Fig. 2-5. 
Table 2: The optimal solution, $\mathrm{K}_{\mathrm{o}}=200$

\begin{tabular}{llllllll}
\hline$\beta$ & $\mathrm{N}_{1}^{*}$ & $\mathrm{Q}_{\mathrm{m} 1}^{*}$ & $\mathrm{~N}_{2}^{*}$ & $\mathrm{Q}_{\mathrm{m} 2}^{*}$ & $\mathrm{~N}_{3}^{*}$ & $\mathrm{Q}_{\mathrm{m} 3}^{*}$ & $\mathrm{~min} \mathrm{E}(\mathrm{TC})$ \\
\hline 0.1 & 6.16683 & 357.339 & 7.02215 & 300.554 & 8.29147 & 355.327 & 8941.81 \\
0.2 & 5.50808 & 336.259 & 6.23161 & 280.790 & 7.29529 & 323.449 & 8954.61 \\
0.3 & 4.99926 & 319.976 & 5.62184 & 265.546 & 6.52868 & 298.918 & 8967.08 \\
0.4 & 4.59296 & 306.975 & 5.13629 & 253.407 & 5.92095 & 279.470 & 8979.22 \\
0.5 & 4.25977 & 296.313 & 4.73954 & 243.489 & 5.42709 & 263.667 & 8991.01 \\
0.6 & 3.98080 & 287.386 & 4.40869 & 235.217 & 5.01765 & 250.565 & 9002.44 \\
0.7 & 3.74335 & 279.787 & 4.12825 & 228.206 & 4.67262 & 239.524 & 9013.52 \\
0.8 & 3.53851 & 273.232 & 3.88732 & 222.183 & 4.37788 & 230.092 & 9024.23 \\
0.9 & 3.35984 & 267.515 & 3.67800 & 216.950 & 4.12321 & 221.943 & 9034.58 \\
1 & 3.20253 & 262.481 & 3.49442 & 212.360 & 3.90099 & 214.832 & 9044.56 \\
\hline
\end{tabular}

Table 3: The optimal solution, $K_{h}=100$

\begin{tabular}{llllllll}
\hline$\beta$ & $\mathrm{N}_{1}^{*}$ & $\mathrm{Q}_{\mathrm{m} 1}^{*}$ & $\mathrm{~N}_{2}^{*}$ & $\mathrm{Q}_{\mathrm{m} 2}^{*}$ & $\mathrm{~N}_{3}^{*}$ & $\mathrm{Q}_{\mathrm{m} 3}^{*}$ & $\mathrm{~min} \mathrm{E}(\mathrm{TC})$ \\
\hline 0.1 & 5.52414 & 336.772 & 6.30517 & 282.629 & 7.48797 & 224.784 & 8942.19 \\
0.2 & 4.88502 & 316.321 & 5.54002 & 263.500 & 6.52647 & 207.476 & 8955.04 \\
0.3 & 4.38068 & 300.182 & 4.93893 & 248.473 & 5.77547 & 193.958 & 8967.54 \\
0.4 & 3.97504 & 287.201 & 4.45747 & 236.437 & 5.17707 & 183.187 & 8979.64 \\
0.5 & 3.64342 & 276.589 & 4.06533 & 226.633 & 4.69202 & 174.456 & 8991.33 \\
0.6 & 3.36841 & 267.789 & 3.74125 & 218.531 & 4.29291 & 167.272 & 9002.60 \\
0.7 & 3.13235 & 260.237 & 3.46725 & 211.706 & 3.87292 & 162.524 & 9013.81 \\
0.8 & 2.92851 & 253.732 & 3.23432 & 205.673 & 3.57988 & 158.092 & 9024.55 \\
0.9 & 2.75984 & 248.215 & 3.02800 & 200.510 & 3.33521 & 154.943 & 9034.99 \\
1 & 2.61253 & 243.281 & 2.86437 & 196.156 & 3.10129 & 152.832 & 9045.11 \\
\hline
\end{tabular}

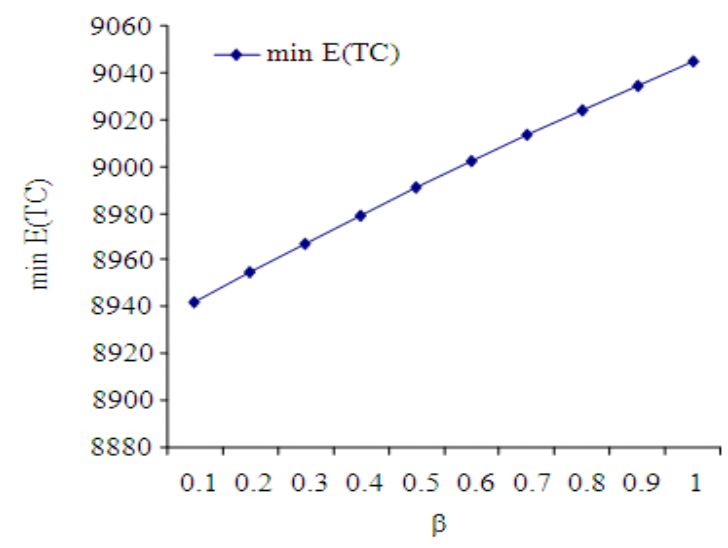

Fig. 4: The relation between $\min \mathrm{E}(\mathrm{TC})$ and $\beta, \mathrm{K}_{\mathrm{o}}=200$

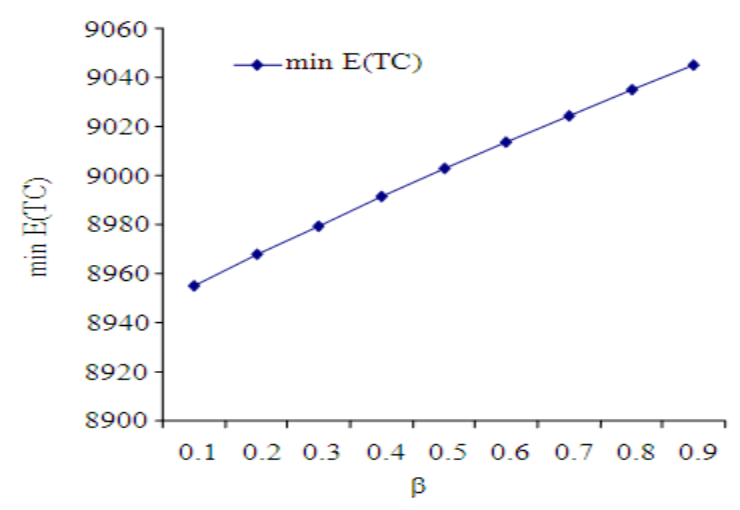

Fig. 5: The relation between $\min \mathrm{E}(\mathrm{TC})$ and $\beta, \mathrm{K}_{\mathrm{h}}=100$

\section{MATERIALS AND METHODS}

The aim of this study is to investigate the periodic review probabilistic multi-item inventory system with zero lead time when the holding cost is a varying function of the inventory cycle. The geometric programming approach is used to determine the optimal inventory cycle and the optimal maximum inventory level which minimize the expected total cost under the expected order cost constraint and under the expected holding cost constraint.

\section{RESULTS AND DISCUSSION}

The basic results of this chapter are.

The minimum annual expected total cost under the expected order cost constraint is given by:

$$
\min E(T C)=\sum_{r=1}^{n}\left[\begin{array}{l}
c_{p r} E\left(D_{r}\right)+c_{o r}\left(\frac{c_{h r} E\left(D_{r}\right)\left(\beta+1-W_{3 r}^{*}\right)}{2 c_{o r}\left(1+W_{3 r}^{*}\right)}\right)^{\frac{1}{\beta+2}}+ \\
\frac{c_{h r}}{2}\left(D_{r}\right) \\
c_{h r} E\left(D_{r}\right) \alpha
\end{array}\right]
$$

And minimum annual expected total cost under the expected varying holding cost constraint is given by: 


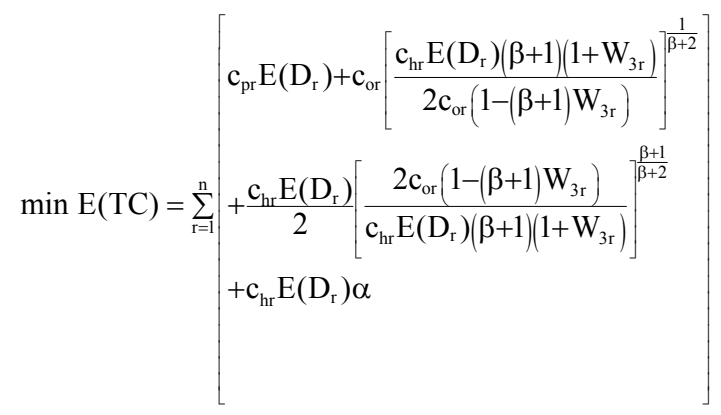

At the end of this paper, a special case of previously published work is added. Also a numerical illustrative example is added with some graphs by using Mathematica program.

\section{CONCLUSION}

We have evaluated the optimal number of periods $\mathrm{N}_{\mathrm{r}}^{*}, \mathrm{r}=1,2, \ldots, \mathrm{n}$ then we deduced the minimum expected total cost min E(TC) of the considered safety stock probabilistic multi-item inventory model. We draw the curves $\mathrm{N}_{\mathrm{r}}^{*}$ and min $\mathrm{E}(\mathrm{TC})$ against $\beta$, which indicate the values of $\mathrm{N}_{\mathrm{r}}^{*}$ and $\beta$ that gives the minimum value of the expected total cost of our numerical example.

\section{REFERENCES}

Abou-El-Ata, Fergany, H.A. and M.F. El-Wakeel, 2002. Probabilistic multi-item inventory model with varying order cost under two restrictions: A geometric programming approach. Intl. J. Product. Econ., 83: 223-231.
Ben-Daya, M. and M. Hariga, 1999. Some stochastic inventory models with deterministic variable lead time. Eur. J. Operat. Res., 42: 51.

Duffin, R.J. and E.L. Peterson, 1974. Constrained minima treated by geometric means. Westinghouse Sci. Paper, 64: 158-129.

Fabrycky, W.J. and J. Banks, 1967. Procurement and Inventory Systems: Theory and Analysis. Reinhold Publishing Corportion, USA.

Fergany, H.A., 2005. Periodic review probabilistic multi-Item inventory system with zero lead Time under constraints and varying order cost. Am. J. Applied Sci., 2: 1213:1217.

Hadley, G. and T.M. Whitin, 1963. Analysis of Inventory Systems. Englewood Cliffs, N.J. Prentice-Hall.

Hariri, A. M. A. and M.O. Abou-El-Ata, 1997. Multiitem production lot size inventory model with varying order cost under a restriction: A geometric programming approach. Product. Plann. Control, 8: 179-182.

Hariri, A.M.A. and M.O. Abou-El-Ata, 1995. Multiitem production lot-size inventory model with varying order cost under a restriction: A Geometric programming approach. Product. Plann. Control., 6: 374-377.

Kotb, K.A.M., 1998. The resources constrained singleitem inventory problem with demand-dependent unit cost. Product. Plann. Control, 9: 700-704.

Taha, H.A., 1997. Operations Research. 6th Edn., Prentice-Hall, INC, Englewood Cliffs, NJ, USA. 